\title{
Effets combinés du biocharbon et du fumier sur les propriétés physico-chimiques d'un sol ferrugineux tropical sous culture de mil en zone semi-aride du Burkina Faso
}

\author{
Désiré Jean Pascal Lompo ${ }^{1 *}$, Lambiénou Yé ${ }^{1}$, Abdoudramane Balboné1, Siélé lbrahima Sori², \\ 1 Université de Dédougou (UDDG), BP 176, Dédougou, Burkina Faso. \\ 2 Bureau national des sols (BUNASOLS), 03 BP 7005 Ouagadougou 03, Burkina Faso \\ *Auteur correspondant : Désiré Jean-Pascal Lompo, Tél. : +226 70278758 \\ E-mail : lompodesire@yahoo.fr
}

Original submitted in on 20 $0^{\text {th }}$ November 2020. Published online at www.m.elewa.org/journals/ on $31^{\text {st }}$ January 2021 https://doi.org/10.35759/JABs.157.3

\section{RESUME}

Objectifs : L'une des contraintes majeures des systèmes de production agricole au Burkina Faso demeure la dégradation des sols. La présente étude menée à Kongoussi (Burkina Faso) avait pour objectif de déterminer les effets du Biocharbon utilisé comme amendement sur le sol et les paramètres agronomiques du mil.

Méthodologie et résultats: Un dispositif en blocs simple comportant 3 traitements en 5 répétitions a été utilisé. Les traitements comprenaient T0 $=$ Fumier + NPK, T1 $=$ Biocharbon + Fumier + NPK, T2 $=$ Biocharbon + NPK. Les matières fertilisantes ont induit une amélioration des paramètres chimiques du sol. Les meilleurs paramètres chimiques du sol ont été observés avec T1 (Biocharbon + Fumier + NPK) qui a permis l'obtention du plus grand rendement grains du mil comparativement à ceux obtenus avec les traitements T0 (Fumier + NPK) et T2 (Biocharbon + NPK) qui étaient statistiquement équivalents.

Conclusions and application des résultats : La combinaison du biocharbon au fumier améliore la fertilité du sol et le rendement du mil. Cela constitue une solution intéressante en vue restaurer et ou maintenir la fertilité des sols, accroitre les rendements des cultures tout en réduisant les effets néfastes des changements climatiques à travers la séquestration du carbone dans le sol. La technologie du Biocharbon constitue une alternative intéressante pour la valorisation des résidus culturaux peu ou pas exploités pour améliorer durablement la qualité des sols et assurer la sécurité alimentaire tout en protégeant l'environnement.

Mots-clés : fertilité des sols ; Biocharbon ; amendement organique ; séquestration du carbone,

\begin{abstract}
Combined effects of biocharbon and manure on physico-chemical properties of a tropical ferruginous soil under millet cultivation in the semi-arid zone of Burkina Faso

Objectives: One of the major constraints of agricultural production systems in Burkina Faso is the low soil fertility. The objective of this study conducted in Kongoussi (Burkina Faso) was to determine the effects of Biochar used as soil amendment in combination with manure on soil fertility and some agronomic parameters of millet.

Methodology and results: A simple block disign with 3 treatments in 5 replicates was used. The randomly distributed treatments included T0 $=$ Manure + NPK, T1 $=$ Biochar + Manure + NPK, T2= Biochar + NPK. The
\end{abstract}


applied fertilizers improved some soil chemical parameters. The best soil chemical parameters were observed with T1 (Biochar + Manure + NPK), which induced the highest millet grain yield as compared to those obtained with T0 (Manure + NPK) and T2 (Biochar + NPK), which were statistically equivalent.

Conclusions and application of findings : The combination of biochar with manure improves soil fertility and millet yield. This is an interesting solution to restore and/or maintain soil fertility, to increase crop yields while reducing the adverse effects of climate change through carbon sequestration into the soil. The Biochar technology is an interesting alternative for the valorization of crop residues little or not well exploited in order to sustainably improve soil quality and food security while protecting the environment.

Keywords: Crop production ; Soil fertility ; Biochar ; organic amendment, carbon sequestration.

\section{INTRODUCTION}

La dégradation des terres est un problème qui se pose avec acuité en Afrique. En effet, les effets combinés des activités agricoles et du changement climatique ont accéléré la dégradation des terres agricoles tant pour les terres arables que pour les pâturages (IFAD, 2019). Plus de la moitié de la superficie des terres agricoles du continent africain serait affectée par le phénomène de dégradation, impactant ainsi négativement les rendements des cultures (OCDE, 2013 ; UNEP, 2015). Au Burkina Faso, l'objectif d'accroitre les productions agricoles pour nourrir une population de plus en plus croissante parait très ambitieux en raison de ce phénomène de dégradation des sols. Le sol naturellement pauvre se dégrade continuellement (Da, 2008). Les terres les plus dégradées se rencontrent dans les régions du Sahel $(57 \%)$, du Nord $(29 \%)$ et du Centre Nord (29\%) (CILSSCSAO, 2008). Ainsi, afin d'assurer les besoins grandissants d'une population toujours croissante, la gestion durable de la fertilité du sol devient une priorité pour tous les pays africains et en particulier le Burkina Faso. Pour ce faire, plusieurs techniques ont donc été adoptées dans les différentes régions du pays afin d'assurer une production agricole durable. Par exemple, dans la région du Centre Nord, la technique du Zaï combiné à l'usage de la matière organique est l'une des principales voies choisies par les producteurs (Da, 2008). Par

\section{MATERIELS ET METHODE}

Zone d'étude: l'étude a été conduite à Kongoussi (Centre-Nord/ Province du Bam) située entre les coordonnées $1^{\circ} 45^{\prime} 60^{\prime \prime}$ et $1^{\circ} 25^{\prime} 39^{\prime \prime}$ de longitude ouest $13^{\circ} 35^{\prime} 00^{\prime \prime}$ et $13^{\circ} 14^{\prime} 22^{\prime \prime}$ de latitude nord. Le climat est de type sub-sahélien caractérisé par l'alternance de deux ailleurs, dans un contexte de changement climatique et dans l'optique de la séquestration du carbone, l'utilisation du biocharbon, technique ancestrale, découvert en Amazonie pour son aptitude à augmenter le stock de carbone dans le sol et à accroitre la productivité des sols serait donc une voie à explorer. Le biocharbon pourrait être une alternative pour lutter contre le réchauffement climatique en conservant le carbone dans le sol et en luttant contre la déforestation par la sédentarisation des agriculteurs (Kimetu et al., 2010; Whitman et al., 2011; Oleszczuk et al., 2012 ; Cayuela et al., 2013). Résidu solide issu de la pyrolyse de biomasses végétales ou animales, le biocharbon attire de plus en plus l'attention des chercheurs partout dans le monde et est considéré comme un amendement qui permettrait d'améliorer la fertilité du sol à travers l'augmentation du taux de matière organique ainsi que l'amélioration de la structure et de la rétention en nutriments et en eau du sol (Laird et al., 2010). De ce fait, il s'avère donc nécessaire d'évaluer la contribution d'une telle technologie sur la productivité des sols au Burkina Faso et en particulier dans les régions arides et semi arides. La présente étude avait pour objectif global d'évaluer les effets du biocharbon sur les paramètres physico-chimiques des sols et les paramètres agronomiques du mil en zone semiaride du Burkina Faso.

saisons : une longue saison sèche de novembre à mai et une courte saison des pluies de juin à octobre (Zombré, 2006) avec un maximum de précipitation enregistré en juin et Août. En 2018 une exceptionnelle pluviosité de $1031 \mathrm{~mm}$ d'eau a été enregistrée. On 
distingue cinq (05) classes de sol dans la commune de Kongoussi : les sols minéraux bruts, les sols à sesquioxydes de fer et lou manganèse, les sols hydromorphes, les sols peu évolués les sols bruns (BUNASOLS, 1995).

Les fertilisants utilisés : du biocharbon de rachis de maïs (Figure 1) finement broyé obtenu par pyrolyse à environ $500^{\circ} \mathrm{C}$ à l'aide de fours locaux (Anderson, 2013 ;
Manka'Aboussi et al., 2019 ; Akoto-Danso et al., 2019) a été utilisé. Le fumier de caprins fréquemment utilisé par la majorité des exploitants de la zone a été considéré dans cette étude. L'engrais minéral utilisé était du NPK (14-23-14). Les caractéristiques chimiques du Biocharbon et du fumier sont présentées dans le Tableau 1.
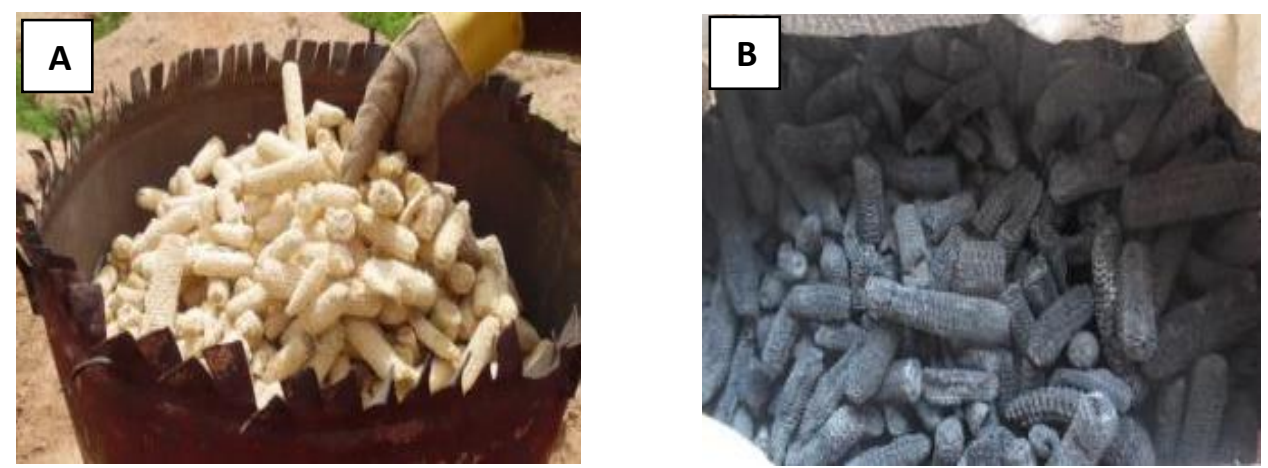

Figure 1 : Rachis de maïs (Image A) et Biocharbon de rachis de maïs ((Image B)

Tableau 1: Caractéristiques chimiques du fumier et du biocharbon utilisés comme amendements

\begin{tabular}{l|c|c|c|c|c|c}
\hline Type d'amendement & $\begin{array}{c}\mathbf{C} \\
\left(\mathbf{g} \cdot \mathbf{k g}^{-1}\right)\end{array}$ & $\begin{array}{c}\mathbf{N} \\
\left(\mathbf{g} \cdot \mathbf{k g}^{-1}\right)\end{array}$ & $\begin{array}{c}\mathbf{P} \\
\left(\mathbf{m g} \cdot \mathbf{k g} \mathbf{-}^{-1}\right)\end{array}$ & $\begin{array}{c}\mathbf{K} \\
\left(\mathbf{m g} \cdot \mathbf{k g}^{-1}\right)\end{array}$ & $\begin{array}{c}\mathbf{C a} \\
\left(\mathbf{m g} \cdot \mathbf{k g}^{-1}\right)\end{array}$ & $\begin{array}{c}\mathbf{S} \\
\left(\mathbf{m g}_{\mathbf{k g}} \mathbf{- 1}\right)\end{array}$ \\
\hline Fumier de caprins & 390 & 22,8 & 424 & 1234 & $\mathrm{nd}$ & $\mathrm{nd}$ \\
Biocharbon de rachis de maïs & 560 & 8,6 & 540 & 1504 & 5,4 & 0,21 \\
\hline
\end{tabular}

$\mathrm{C}:$ Carbone, $\mathrm{N}$ : Azote, $\mathrm{P}:$ Phosphore ; $\mathrm{K}:$ Potassium ; $\mathrm{Ca}:$ Calcium ; $\mathrm{S}:$ Souffre ; nd : non déterminé

Dispositif expérimental : le dispositif mis en place est un dispositif en blocs simples comportant 03 traitements en 05 répétitions $\mathrm{TO}=$ Fumier+ NPK, $\mathrm{T} 1=$ Biocharbon + Fumier+ NPK et T2= Biocharbon+ NPK. Les traitements ont été répartis de façon randomisée dans chaque bloc. Les parcelles élémentaires mesuraient $4 \mathrm{~m} \times 4 \mathrm{~m}\left(16 \mathrm{~m}^{2}\right)$ et étaient distantes de $1 \mathrm{~m}$.

Les opérations culturales : la préparation du sol a consisté à réaliser sur chaque parcelle élémentaire, des trous de Zaï ou poquets de $20 \mathrm{~cm}$ de diamètre et $15 \mathrm{~cm}$ de profondeur, espacés de $0.8 \mathrm{~m}$ entre eux. Les applications du fumier et du biocharbon ont été réalisées dans les trous de zaï à des doses respectives de $250 \mathrm{~g} /$ trou de Zaï (2,5t/ha) et $200 \mathrm{~g} /$ trou de Zaï $(2,5 \mathrm{t} / \mathrm{ha})$ (Figure 2). Le mil (Pennisetum glaucum $(\mathrm{L})$ r. Br) variété MISSARI 1 a été utilisé comme culture test (Figure 3). Le sarclage a été fait 15 jours après semis au moment de l'application du NPK (14-23-14). 

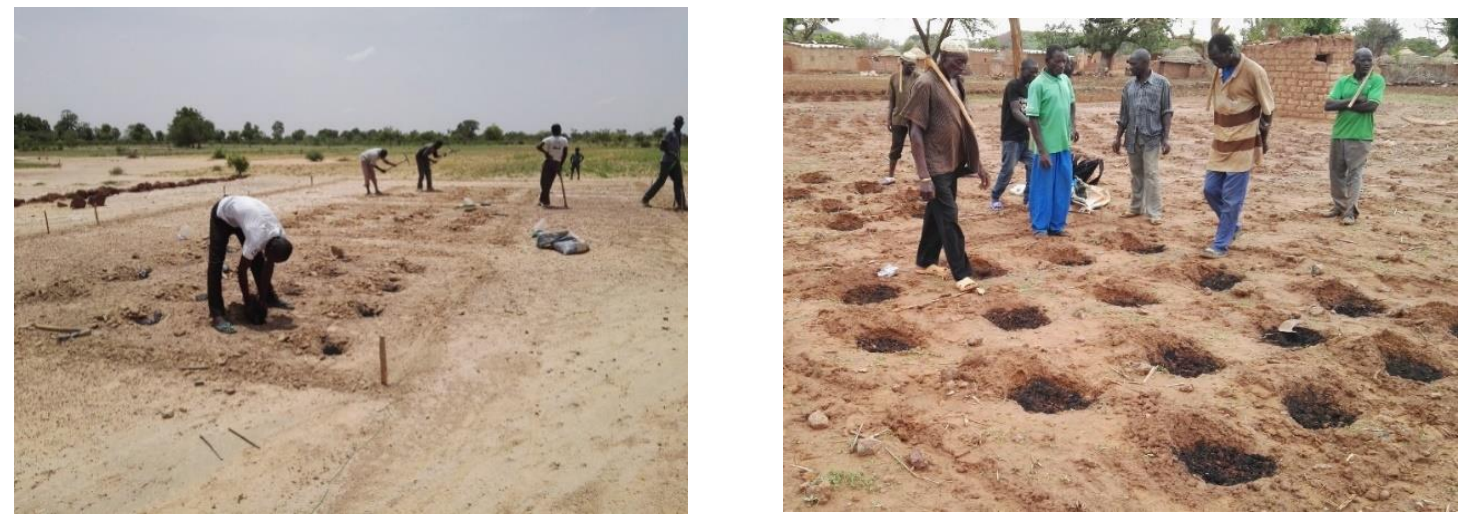

Figure 2 : Illustration des trous de zaï avec le biocharbon et le fumier appliqués

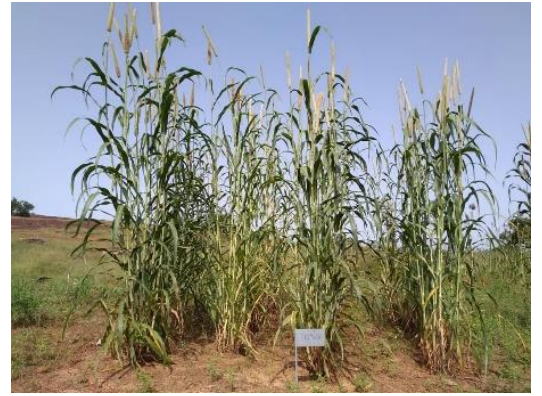

TO : Fumier+NPK

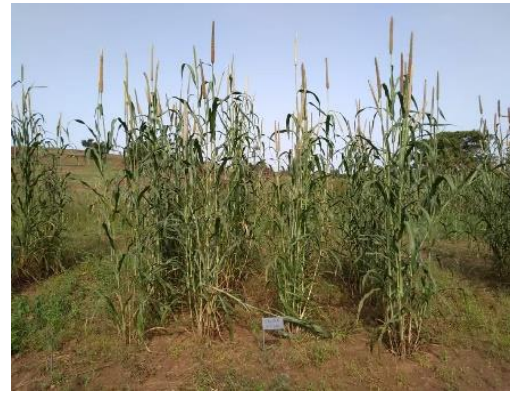

T1 : Biocharbon+Fumier+NPK

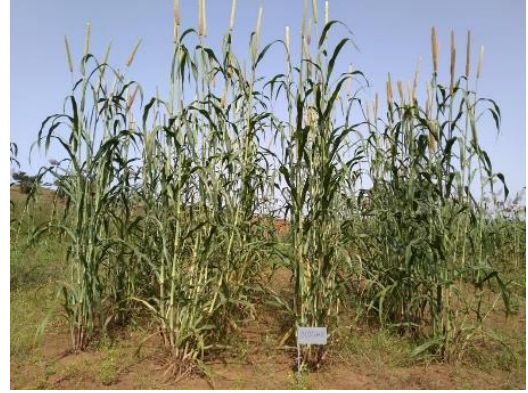

T2 : Biocharbon+NPK

Figure 3: Illustration de l'état des plants de mil pendant la phase de remplissage des grains en fonction des traitements T0, T1 et T2.

Paramètres mesurés/déterminés: la hauteur des plants à 27, 34, 41 et 48 jours après semis (JAS), le diamètre des plants à $48 \mathrm{JAS}$, le rendement grain du mil, les caractéristiques physico-chimiques des sols ont été les paramètres évalués. Les échantillons de sol ont été prélevés dans l'horizon 0-20 cm, séchés à l'air libre et à l'ombre puis tamisés à $2 \mathrm{~mm}$ pour les analyses au laboratoire. Les paramètres analysés ont été la granulométrie 3 fractions par la méthode à la Pipette de Robinson (AFNOR, 1987), le pHeau déterminé à l'aide d'un pH-mètre avec un rapport sol/eau de 1/2,5 (BUNASOLS, 1987), le carbone organique déterminé par la méthode de Walkey Black (1934), la capacité d'échange cationique (CEC) déterminée par la méthode

\section{RESULTATS}

Effets des traitements sur les propriétés physicochimiques des sols: les résultats montrent que les teneurs en éléments nutritifs des sols traités et des sols initiaux sont restés similaire au seuil de probabilité de $5 \%$ (Tableau 2). Cependant, la teneur en carbone dans les sols sous traitements a augmenté sauf pour le traitement Fumier+NPK (T0). Les traitements
METSON (AFNOR, 1987), l'azote total (N) par la méthode Kjeldahl (BUNASOLS, 1987), le phosphore total $(P)$ et le potassium total $(K)$ déterminés par minéralisation avec l'acide sulfurique salsalique, puis le $\mathrm{P}$ a été déterminé par l'auto analyseur et le $\mathrm{K}$ par le photomètre à flamme. La densité apparente a été déterminée en faisant le rapport poids/volume (BUNASOLS, 1987).

Analyse statistique des données: l'analyse de la variance (ANOVA) a été effectuée à l'aide du logiciel GENSTAT version 12.1. Le test de Student NewmanKeuls a été utilisé pour les comparaisons multiples, afin de séparer les moyennes au seuil de $5 \%$.

Biocharbon+Fumier+NPK (T1) et Biocharbon+NPK (T2) ont entrainé des augmentations de $28 \%$ et $13 \%$ respectivement contre une baisse de 1,7\% occasionné par le traitement Fumier+NPK (T0). Pour la teneur en azote total, on a observé une baisse de $23 \%$ et $4 \%$ respectivement pour les traitements Fumier+NPK (T0) et Biocharbon+NPK (T2). Par contre, Le traitement 
Biocharbon+Fumier+NPK a induit une augmentation de la teneur en azote total du sol qui est de $11 \%$. Les mêmes observations ont été faites pour la teneur en potassium total du sol. Des baisses de $19 \%$ et $9 \%$ ont été observé avec les traitements Fumier+NPK (T0) et Biocharbon+NPK (T2) contre une augmentation de 9,6\% avec le traitement Biocharbon+Fumier+NPK (T1). Tous les traitements ont induit une augmentation de la teneur en phosphore total du sol. Les augmentations sont de l'ordre de $9 \%, 27 \%$ et $17 \%$ respectivement pour les traitements Fumier+NPK

Biocharbon+Fumier+NPK (T1) et Biocharbon+NPK (T2). Le $\mathrm{pH}$ des sols soumis aux différents traitements a augmenté comparativement au sol initial. Cette augmentation a été hautement significatif $(p<0,01)$. La plus grande valeur a été obtenue avec le traitement
Biocharbon+NPK (T2). Dans ce traitement (T2), le pH varie de 0,95 unité contre 0,65 unité pour le traitement Fumier+NPK (T0) et 0,91 unité pour le traitement Biocharbon+Fumier+NPK (T1). La capacité d'échange cationique (CEC) a significativement augmenté dans tous les sols traités $(p<0,05)$. Les augmentations sont respectivement de $55,6 \%, 59 \%$ et $52,4 \%$ pour les traitements Fumier+NPK Biocharbon+Fumier+NPK (T1) et Biocharbon+NPK (T2). La densité apparente a diminué dans les sols traités au biocharbon (T1 et T2) et augmenté dans le traitement ne contenant pas le biocharbon (T0). La densité apparente est passée de 1,63 à 1,648 pour le traitement Fumier+NPK (T0); de 1,63 à 1,62 pour le traitement Biocharbon+Fumier+NPK (T1) et de 1,63 à 1,61 pour le traitement Biocharbon+NPK (T2). 
Lompo et al., J. Appl. Biosci. 2021 Effets combinés du biocharbon et du fumier sur les propriétés physico-chimiques d'un sol ferrugineux tropical sous culture de mil en zone semi-aride du Burkina Faso.

Tableau 2 : caractéristiques physico-chimiques des sols avant et après récolte en fonction des traitements. Chaque valeur représente la moyenne $(n=5)$ suivies de l'écart type

\begin{tabular}{|c|c|c|c|c|c|c|c|c|c|c|c|}
\hline & Traitement & $\begin{array}{c}\text { Argile } \\
(\%)\end{array}$ & $\begin{array}{c}\text { Limon } \\
(\%)\end{array}$ & $\begin{array}{c}\text { Sable } \\
(\%)\end{array}$ & $\begin{array}{l}\text { Corg } \\
(\mathrm{g} / \mathrm{kg})\end{array}$ & $\begin{array}{l}\text { Ntotal } \\
(\mathrm{g} / \mathrm{kg})\end{array}$ & $\begin{array}{l}\text { Ptotal } \\
(\mathrm{mg} / \mathrm{kg})\end{array}$ & $\begin{array}{c}\text { Ktotal } \\
(\mathrm{mg} / \mathrm{kg})\end{array}$ & pHeau & $\begin{array}{c}\text { Densité } \\
\text { apparente }\end{array}$ & $\begin{array}{c}\text { CEC } \\
(\mathrm{mg} / \mathrm{kg})\end{array}$ \\
\hline \multirow[t]{2}{*}{$\begin{array}{l}\text { Avant la } \\
\text { culture }\end{array}$} & - & $\begin{array}{l}7,18 \\
(1,3)\end{array}$ & $11,4(4,9)$ & $\begin{array}{l}81,4 \\
(6,2)\end{array}$ & $\begin{array}{l}0,58 \\
(0,2)\end{array}$ & $\begin{array}{c}0,05 \\
(0,008)\end{array}$ & $\begin{array}{l}199,7 \\
(76,5)\end{array}$ & $\begin{array}{l}616,8 \\
(68,5)\end{array}$ & $\begin{array}{c}5,5(0,27) \\
a\end{array}$ & $1,63(0,02) a$ & $\begin{array}{c}2,56(0,72) \\
a\end{array}$ \\
\hline & TO & $\begin{array}{c}6,7 \\
(0,91)\end{array}$ & $\begin{array}{l}10,47 \\
(6,8)\end{array}$ & $\begin{array}{l}82,8 \\
(7,7)\end{array}$ & $\begin{array}{l}0,57 \\
(0,3)\end{array}$ & $\begin{array}{c}0,04 \\
(0,01)\end{array}$ & $\begin{array}{l}220,6 \\
(85,8)\end{array}$ & $\begin{array}{l}495,3 \\
(84,1)\end{array}$ & $\begin{array}{c}6,1(0,33) \\
b\end{array}$ & $1,65(0,025) b$ & $5,77(1,54)$ \\
\hline \multirow[t]{2}{*}{$\begin{array}{l}\text { Après } \\
\text { récolte }\end{array}$} & $\mathrm{T} 1$ & $\begin{array}{c}6,2 \\
(1,3)\end{array}$ & $11,3(6,7)$ & $\begin{array}{l}82,5 \\
(8,2)\end{array}$ & $\begin{array}{c}0,81 \\
(0,19)\end{array}$ & $\begin{array}{c}0,05 \\
(0,006)\end{array}$ & $\begin{array}{l}276,2 \\
(80,1)\end{array}$ & $\begin{array}{l}682,3 \\
(65,4)\end{array}$ & $\begin{array}{c}6,4(0,2) \\
b\end{array}$ & $1,62(0,03) a b$ & $6,22(1,26)$ \\
\hline & T2 & $\begin{array}{c}6,2 \\
(1,2)\end{array}$ & $\begin{array}{l}10,45 \\
(7,01)\end{array}$ & $\begin{array}{l}83,3 \\
(7,2)\end{array}$ & $\begin{array}{l}0,67 \\
(0,2)\end{array}$ & $\begin{array}{c}0,04 \\
(0,006)\end{array}$ & $241,4(65)$ & $\begin{array}{l}560,8 \\
(68,53)\end{array}$ & $\begin{array}{c}6,4(0,33) \\
b\end{array}$ & $1,61(0,023) a b$ & $5,38(1,31)$ \\
\hline \multicolumn{2}{|c|}{$\begin{array}{l}\text { Probabilité (5\%) } \\
\text { CV (\%) }\end{array}$} & $\begin{array}{l}0,061 \\
24,6\end{array}$ & $\begin{array}{l}0,49 \\
72,3\end{array}$ & $\begin{array}{c}0,085 \\
10,7\end{array}$ & $\begin{array}{l}0,06 \\
40,3\end{array}$ & $\begin{array}{l}0,32 \\
25,2\end{array}$ & $\begin{array}{l}0,17 \\
40,1\end{array}$ & $\begin{array}{c}0,082 \\
7\end{array}$ & $\begin{array}{c}<0,01 \\
6,1\end{array}$ & $\begin{array}{c}0,011 \\
18\end{array}$ & $\begin{array}{l}0,02 \\
221\end{array}$ \\
\hline
\end{tabular}

$T 0=$ Fumier+NPK $; T 1=$ Biocharbon+fumier+NPK ; T2 = Biocharbon+NPK; CV : coefficient de variation. Les valeurs affectées d'une même lettre dans la même colonne ne sont pas significativement différentes à $P=5 \%$ selon test de Student Newman Keuhl 
Lompo et al., J. Appl. Biosci. 2021 Effets combinés du biocharbon et du fumier sur les propriétés physico-chimiques d'un sol ferrugineux tropical sous culture de mil en zone semi-aride du Burkina Faso.

Effets des traitements sur les paramètres agronomiques de mil : les résultats montrent qu'il n'y a pas de différences significatives entre les traitements pour les hauteurs des plants de mil $(p \geq 0,05)$. Par contre, ils révèlent une différence significative entre les traitements pour le diamètre des plants $(p<0,05)$. Le diamètre des plants le plus élevé $(1,8 \mathrm{~cm})$ a été obtenu avec le traitement T1 (Biocharbon+ Fumier+ NPK). Les traitements T0 (Fumier+ NPK) et T2 (Biocharbon+ NPK) ont induit $1,7 \mathrm{~cm}$ et $1,6 \mathrm{~cm}$ de diamètre respectivement (Tableau 3).

Tableau 3: Variations des hauteurs et diamètres des plants de mil en fonction des traitements. Les valeurs représentent les moyennes $(n=5)$ suivi de l'écart type

\begin{tabular}{l|c|c|c|c|c}
\hline \multicolumn{1}{c|}{ Traitements } & $\begin{array}{c}\text { Hauteur } \\
\text { 27 JAS } \\
(\mathbf{c m})\end{array}$ & $\begin{array}{c}\text { Hauteur } \\
\text { 34 JAS } \\
(\mathbf{c m})\end{array}$ & $\begin{array}{c}\text { Hauteur } \\
\text { 41 JAS } \\
(\mathbf{c m})\end{array}$ & $\begin{array}{c}\text { Hauteur } \\
\text { 48 JAS } \\
(\mathbf{c m})\end{array}$ & $\begin{array}{c}\text { Diamètre } \\
\text { 48 JAS } \\
(\mathbf{c m})\end{array}$ \\
\hline T0 : Fumier + NPK & $62,3(28)$ & $90,9(40,4)$ & $143,3(46,5)$ & $191,2(45)$ & $1,7(0,1) \mathrm{a}$ \\
T1: Biocharbon + Fumier + NPK & $73,6(27,6)$ & $112,9(43,7)$ & $179,2(55)$ & $230,3(50,3)$ & $1,8(0,1) \mathrm{b}$ \\
T2: Biocharbon + NPK & $57,0(26,1)$ & $82,5(35,2)$ & $141,1(51,5)$ & $198(50,1)$ & $1,8(0,2) \mathrm{a}$ \\
\hline Probabilité (5\%) & 0,065 & 0,05 & 0,056 & 0,067 & 0,022 \\
CV (\%) & 42,1 & 41,1 & 33,9 & 21,2 & 4 \\
\hline
\end{tabular}

JAS : jour après semis ; CV : coefficient de variation ; Les chiffres affectés d'une même lettre dans la même colonne ne sont pas significativement différents à $P=5 \%$ selon le test de Student Newman Keuhl

Concernant les rendements grains du mil, les résultats montrent une différence significative entre les traitements $(p<0,05)$. Le rendement grain obtenu avec le traitement combinant le biocharbon et le fumier (T1) est statistiquement supérieur à ceux des traitements Fumier+ NPK (T0) et Biocharbon+ NPK (T2). Qui sont statistiquement équivalents et qui ont donné respectivement $1498 \mathrm{~kg} \cdot$ ha $^{-1}$ et $1535 \mathrm{~kg} \cdot$ ha-1 $^{-1}$ (Tableau 4).

Tableau 4 : Variation du rendement grain du mil en fonction des traitements. Les valeurs représentent les moyennes $(\mathrm{n}=5)$ suivi de l'écart type

\begin{tabular}{l|l}
\hline Traitements & \multicolumn{1}{c}{ Rendement grains (Kg/ha) } \\
\hline Fumier+ NPK (T0) & $1498^{\mathrm{a}}(453,9)$ \\
Biocharbon+ Fumier+ NPK (T1) & $2000^{\mathrm{b}}(411,7)$ \\
Biocharbon+ Fumier+ NPK (T2) & $1535^{\mathrm{a}}(442)$ \\
\hline Probabilité (5\%) & 0,04 \\
\hline
\end{tabular}

Les chiffres affectés d'une même lettre dans la même colonne ne sont pas significativement différents à $P=5 \%$ selon test de Student Newman Keuhl

\section{DISCUSSION}

Effet des traitements sur des propriétés physicochimiques des sols: les caractéristiques physicochimiques du sol après récolte sont meilleures avec les traitements T1 (Biocharbon+ Fumier+ NPK). Des résultats similaires ont été observés par Lompo et al. (2020). La combinaison du biocharbon et du fumier a induit une plus grande augmentation du carbone organique du sol. Cela est fonction de la richesse en carbone du biocharbon $(56,03 \%)$ et du fumier $(39,63 \%)$ mais aussi et surtout au caractère récalcitrant à la minéralisation du carbone contenu dans le biocharbon (Gomez et al., 2014). Les tendances observées montrent une plus grande augmentation de la teneur en phosphore du sol avec le traitement T1 (Biocharbon+ Fumier+ NPK) suivi du T2 (Biocharbon+ NPK) et du T0
(Fumier+ NPK). Ces résultats seraient dus à l'effet de l'augmentation du pH du sol. En effet, le Phosphore est en général immobilisé aux $\mathrm{pH}$ inférieurs à 6 et l'augmentation du pH du sol sous Biocharbon a eu des effets positifs sur la biodisponibilité du phosphore (Brady et Weil, 2002). De plus, la grande surface d'absorption du phosphore permet de minimiser les pertes par lixiviation du phosphore (Zhang et al., 2010). La teneur en azote des sols traités avec le biocharbon et le fumier (T1) a augmenté contrairement aux autres traitements et ceux malgré les exportations et les lixiviations. Cela serait lié au fait que l'azote provenant de l'engrais minéral (NPK) et de la minéralisation du fumier est maintenu dans le sol par les particules de biocharbon (Alling, 2014 ; Kamman et al., 2015). La plus grande 
valeur du $\mathrm{pH}$ est obtenue avec le traitement T2 (Biocharbon+ NPK). Le biocharbon appliqué seul au sol relève plus les valeurs de $\mathrm{pH}$ que lorsqu'il est appliqué en combinaison avec le fumier ou lorsque le fumier est appliqué seul comme amendement. Ces résultats corroborent ceux obtenus par Lompo et al. (2020). L'augmentation du $\mathrm{pH}$ des sols traités serait due à l'apport de cations basiques par le biocharbon (Frimpong Manso et al., 2019). Les résultats ont montré que la densité apparente des sols soumis aux traitements T1 (Biocharbon+ Fumier+ NPK) et T2 (Biocharbon+ NPK) a diminué contrairement aux sols soumis au traitement TO (Fumier+ NPK). Une diminution de la densité apparente du sol entraine l'augmentation de la porosité et l'aération du sol et peut avoir un effet positif sur les racines et la respiration microbienne (N'dayegamiye, 2007). Par ailleurs, Brodowski et al. (2006) affirment que le biocharbon, en augmentant la porosité du sol, permet l'approvisionnement en oxygène du sol. Enfin, l'augmentation de la teneur en CEC des sols traités pourrait s'expliquer par l'augmentation de la teneur en matière organique et le $\mathrm{pH}$ du sol. En effet,

\section{CONCLUSION ET APPLICATION DES RÉSULTATS}

L'évaluation de l'effet du biocharbon utilisé comme amendement sur les propriétés physico-chimiques du sol et les paramètres agronomiques du mil dans la zone semi-aride du Burkina Faso, a fait l'objet de notre étude. Le biocharbon associé au fumier assure une bonne gestion des éléments minéraux dans le sol et cela se

\section{REMERCIEMENTS}

Ce travail a été réalisé grâce au financement de la Mairie de LUDWIGSBURG (en Allemagne) que nous remercions sincèrement. Nous remercions également les acteurs suivants pour leurs soutiens multiformes : la

\section{REFERENCES}

AFNOR, 1987. Les dossiers de la normalisation, - ISSN 0297-4827, ISBN 2-12-484221-8 : 130,85 F HT, $159 \mathrm{p}$.

Akoto-Danso, E. K., Manka'abusi, D., Steiner, C., Werner, S., Haering, V., Lompo, D.J.P., Nyarko, G., Marschner, B., Drechsel, P., Buerkert, A., (2019). Nutrient flows and balances in intensively managed vegetable production of two West African cities. Journal of plant nutrition and soil science, 1-15. DOI: 10.1002/jpln.201800339

Alling V., Hale S. E., Martinsen V., Mulder J., Smebye A., Breedveld G. D., et Cornelissen G., 2014.
Mbonigaba (2007) et Mze (2008) ont montré qu'une augmentation de la matière organique et du $\mathrm{pH}$ du sol entraine une augmentation de la CEC du sol.

Effet des traitements sur les paramètres agronomiques du mil : l'amendement du sol avec le biocharbon a eu un effet positif le diamètre et le rendement grain du mil. Le rendement grains le plus élevé a été obtenu avec le traitement T1 (Biocharbon+ Fumier+ NPK) alors que le traitement TO (Fumier+ NPK) a donné le plus faible rendement grains. Cela serait dû au fait que l'association du biocharbon et du fumier a permis une meilleure gestion des éléments minéraux dans le sol et par conséquent, a occasionné une plus grande productivité du mil. Ces résultats sont conformes aux résultats de Liu et al. (2012) sur la culture du maïs et ceux de Lompo et al. (2020) sur la culture de sorgho. Ces résultats suggèrent qu'il conviendra d'adopter une pratique de fertilisation combinant le biocharbon, le compost ou le fumier et les engrais minéraux azotés afin d'assurer une augmentation de la production agricole de façon durable.

traduit par un bon développement des cultures de mil. De ce fait, la combinaison du biocharbon et du fumier comme amendement des sols est une nouvelle alternative pour une amélioration de la fertilité des sols au Burkina Faso et pour l'atteinte de la sécurité alimentaire.

Mairie de Kongoussi, l'ONG AZND de Kongoussi, le BUNASOLS, les producteurs et les populations de Bamvillage, Bogonam, Kiella, Kouka, Sankondé et Woussé.

The role of biochar in retaining nutrients in amended tropical soils. Journal of Plant Nutrition and Soil Science. 177: 671-680.

Anderson P.S., 2013. Affordable Biochar Production Options Small Cookstoves, Medium Barrels, and Some Large Devices. Presentation to First Midwest Biochar Conference, 14 June 2013, Champaign, IL. 50p. http://www.biochar.illinois.edu

Brady N.C., et Weil R.R., 2002. Biochar and its effects on plant productivity and nutrient cycling: a meta-analysis. GCB Bioenergy, doi : $\underline{10.1111 / \mathrm{gcbb} .12037}$ 
Brodowski S, John B, Flessa H, and Amelung W., 2006. Aggregate-occluded black carbon in soil. European journal of soil science, $57,539-5$.

BUNASOLS, 1987. Méthode d'analyse physique et chimique des sols, eaux et plantes. Document technique $\mathrm{N}^{\circ} 3,286 \mathrm{p}$.

BUNASOLS, 1995. Étude morphopédologique de la province du Barn, échelle 1150.000, vol. 1 rapport technique $n^{\circ} 97,95 p$.

Cayuela ML., Monedero M., Roig A., Hanley K., Enders A et Lehmann J., 2013. Biochar and denitrification in soils: when, how much and why does biochar reduce $\mathrm{N}_{2} \mathrm{O}$ emissions? Scientific Reports 3.

CILSS-CSAO, 2008. Profil sécurité alimentaire Burkina Faso. Rapport final Ministère des affaires étrangères et européen Burkina Faso. www.food.security.net

Da C. E. D., 2008. Impact des techniques de conservation des eaux et des sols sur le rendement du sorgho au Centre-Nord du Burkina Faso. Cahiers d'Outre-Mer, 61, 241242, 99-110. https://doi.org/10.4000/com.3512

Frimpong Manso, E., Nartey, E.K., Adjadeh T.A., Darko D.A., Lawson, I.Y.D. and Amoatey, C.A., 2019. Use of Corn Cob and Rice Husk Biochars as Liming Materials in Acid Soils. West African Journal of Applied Ecology, vol. 27(2), 32-50.

Gomez J. D., Denef K., Stewart C. E., Zheng J., et Cotrufo M. F., 2014. Biochar addition rate influences soil microbial abundance and activity in temperate soils. European Journal of Soil Science. $\quad 65$ : 28-39. http://unt.unice.fr/uoh/degsol/fertilitephysique.php

IFAD (International Fund for Agricultural Development), 2019. Creating opportunity for rural youth, Rural Development Report, Rome, Italy.

Kamman, C.I., Schmidt, H.-P., Messerschmidt, N., Linsel, S., Steffens, D., Müller, C., Koyro, H.-W., Conte,P., Joseph, S., 2015. Plant growth improvement mediated by nitrate Capture in cocomposted biochar. Sci Rep 5, Article number 11080. https://doi.org/10.1038/srep11080

Kimetu JM and Lehmann J, 2010. Stability and stabilization of biocharbon and green manure in : soil with different organic carbon contents. Australian J of Soil Research 48 : 577- 585

Laird D.A., Fleming P., Davis D.D., Horton R., Wang B., Karlen D.L., 2010. Impact of biochar amendements on the quality of a typical
Midwestern agricultural soil. Geoderma 158 (September), p 443-449.

Liu, J., Schulz, H., Brandl, S., Miehtke, H., Huwe, B., \& Glaser, B., 2012. Short-term effect of biochar and compost on soil fertility and water status of a Dystric Cambisol in NE Germany under field conditions. Journal of Plant Nutrition and Soil Science 175, 698-707

Lompo D.J.P., Balbone A., Yé L., Nacro H.B., 2020. Effets du Biocharbon utilisé comme amendement sur les propriétés physicochimiques des sols et les paramètres agronomiques du Sorgho dans la zone semiaride du Burkina Faso. Science et technique, Sciences naturelles et appliquées, Spécial hors-série $n^{\circ} 5$, pp 387-398.

Manka'abusi, D., Steiner, C., Akoto-Danso, E.K, Lompo, D.J.P., Haering, V., Werner, S., Marschner, B., Buerkert, A, (2019). Biochar application and wastewater irrigation in urban vegetable production of Ouagadougou, Burkina Faso. Nutrient cycling in agroecosystems, https://doi.org/10.1007/s10705-019-09969$\underline{0(0123456789)}$

Mbonigaba M.J.J., 2007. Étude de l'impact des composts à base de biomasse végétale sur la dynamique des indicateurs physico-chimiques, chimiques et microbiologiques de la fertilité des sols : application sur trios sols acides tropicaux du Rwanda. Thèse de doctorat, FUSAGx, Gembloux, 243 p.

Mze S.P., 2008. Influence d'apports en matières organiques sur l'activité biologique et la disponibilité du phosphore dans deux sols de la région des grands lacs d'Afrique. Thèse de doctorat. Faculté Universitaire des Sciences Agronomiques de Gembloux. Gembloux, $240 \mathrm{p}$.

N'dayegamiye, A., 2007. Le travail du sol: une importante régie agricole. Le producteur de lait Québécois, $39-42$. https://www.agrireseau.net/documents/73623// e-travail-du-sol-une-importante-regie-agricole

NEPAD (New Partnership for Africa's Development), 2013. Les agricultures africaines, Transformation et perspectives, Johannesburg, South Africa.

OCDE, 2013. Peuplement, marché et sécurité alimentaire, cahiers de l'Afrique de l'ouest, ed. de l'OCDE

Oleszczuk P., Hale S., Lehmann J., Cornelissen G., 2012. Activated carbon and biochar 
amendments decrease pore-water concentrations of polycyclic aromatic hydrocarbons (PAHs) in sewage sludge. Bioresource Technology 111: 84-91.

Spokas, K.A., 2010. Review of the stability of biochar in soils : predictability of $\mathrm{O}: \mathrm{C}$ molar ratio. Carbon Management (2010) 1(2), 289-303. DOl : $10.4155 / \mathrm{cmt} .10 .32$

UNEP, 2015. The economics of Land Degradation in Africa : Benefits of Action outweigh the costs, Nairobi, Ecosystem Services Economics Units, UNEP Division of Environmental Policy Implementation.

Walkley, A.J. and Black, I.A. (1934) Estimation of soil organic carbon by the chromic acid titration method. Soil Sci. 37, 29-38.

Whitman T, Nicholson CF., Torres D. and Lehmann J., 2011. Climate change impact of biochar cook stoves in Western Kenyan farm households: System dynamics model analysis. Environmental Science and Technology. Environ. Sci. Technol. 2011, 45, 8, 3687-3694. https://doi.org/10.1021/es103301k

Zhang W., Niu J., Morales VL., Chen X., Hay AG., Lehmann J. and Steenhuis TS., 2010. Effect of biochar amendment on yield and methane and nitrous oxide emissions from a rice paddy from Tai Lake plain, China. Agriculture, Ecosystems and Environment. 139:469-475.

Zombré P. N., 2006 : Evolution de l'occupation des terres et localisation des sols nus dans le Centre-Nord du Burkina Faso. Télédétection, 6, 4, 285-297. 